\title{
Weak Gravitational Lensing by Stringy Black Holes
}

\author{
Wajiha Javed, ${ }^{1, *}$ Muhammad Bilal Khadim, ${ }^{2, \dagger}$ Jameela Abbas, $^{3,}{ }^{\ddagger}$ and Ali Övgün ${ }^{4,5}, \S$ \\ ${ }^{1}$ Division of Science and Technology, University of Education, Township-Lahore, Pakistan \\ ${ }^{2}$ Division of Science and Technology, University of Education, Township-Lahore, Pakistan. \\ ${ }^{3}$ Department of Mathematics, University of Education, \\ Township, Lahore-54590, Pakistan. \\ ${ }^{4}$ Instituto de Física, Pontificia Universidad Católica de Valparaíso, Casilla 4950, Valparaíso, Chile. \\ ${ }^{5}$ Physics Department, Arts and Sciences Faculty, Eastern Mediterranean University, Famagusta, North Cyprus via Mersin 10, Turkey. \\ (Dated: December 1, 2019)
}

\begin{abstract}
In this paper, we discuss the weak gravitational lensing in the context of stringy black holes. Initially, we examine the deflection angle of photon by charged stringy black h ole. For this desire, we compute the Gaussian optical curvature and implement the Gauss-Bonnet theorem to investigate the deflection a ngle for s pherically $b$ alanced s pacetime of s tringy b lack $h$ ole. We a lso a nalyze the influence of plasma m edium in the w eak g ravitational lensing for s tringy black $h$ ole. Moreover, the graphical impact of coupling constant $\alpha$, impact parameter $b$, black hole charge $Q$ on deflection angle by charged stringy black hole has been studied in plasma as well as non-plasma medium.
\end{abstract}

PACS numbers: 95.30.Sf, 98.62.Sb, 97.60.Lf

Keywords: Weak gravitational lensing; Stringy black hole; Deflection angle; Gauss-Bonnet theorem

\section{INTRODUCTION}

Einstein in 1916 announced about the presence of the gravitational lensing as well as gravitational waves as a part of the theory of general relativity (GR). His theory depends on many experimental and observational tests. In accordance with his theory, the force of gravity due to the fact of curvature in spacetime and gravitational waves [1] are ripples in the fabric of a universe which are obtained by the clashing of the gigantic objects such like black holes (BHs). The phenomenon in which gravity deflects light is referred as gravitational lensing. Gravitational lensing is very useful method to understand dark matter, galaxies, and universe. After the first observation of Eddington, so many works have been done on gravitational lensing for black holes, wormholes and other objects. The topic of gravitational lensing take a great attraction. Therefore, the gravitational lensing is categories into two ways; strong gravitational lensing and other one is a weak gravitational lensing. The strong gravitational lensing tells us about the magnification, position and time delays of the images by B Hs. Strong lensing phenomena also need in many cases which provide further information from experimental frame of reference to see the other different objects such as monopoles [2], boson stars [3-5], fermion stars [6, 7] etc. On the other hand the weak gravitational lensing give a method to find the mass of astronomical objects without demanding to their formation or dynamical states. Weak lensing also distinguish between dark energy and modified gravity and also examine the cause of rapid expansion in the universe. The gravitational lensing is by means of the distribution of matter among the source and observer, that is qualified by deflection of light from the source to the observer also the deflection of light is one of the prediction of Einstein's theory [8-34]. The idea first given by German astronomer Johan Georg Van Soldner 1801 in the background of Newtonian theory. In 1924 Chwolson and Einstein in 1936 derived the exact calculations of deflection of light in the framework of GR. Regarding to this, Gibbons and werner have investigated the deflection angle of light for asymptotically flat static BHs [35] by using Gauss-Bonnet theorem which is defined as:

$$
\hat{\alpha}=-\iint_{D_{\infty}} \mathcal{K} d \tilde{\sigma}
$$

where $\mathcal{K}$ stand for Gaussian curvature and $d \tilde{\sigma}$ is a surface element of optical metric. After that, Werner expanded this strategy for stationary BHs [36]. Arakida and Kasai [37] have analyzed the engagement of cosmological constant on deflection of light also its status with in the cosmological lens equation. In recent times, Bisnovatyi-Kogan and

\footnotetext{
*Electronic address: wajiha.javed@ue.edu.pk; wajihajaved84@yahoo.com

${ }^{\dagger}$ Electronic address: blaljutt723@gmail.com

‡Electronic address: jameelaabbas30@gmail.com

§Electronic address: ali.ovgun@pucv.cl
} 
Tsupko [38] have showed that the deflection angle in the homogeneous plasma is differ from the deflection angle in a vacuum, in addition that the deflection angle in plasma medium found on the photon frequency. Additionally, this procedure has been prolonged to the wormhole geometries and non-asymptotically flat spacetimes having topological defects [39-46].

According to GR, spacetime singularities rise various issues, both scientific and physical [47, 48], by applying the nonlinear electrodynamics it is reasonable to solve these singularities by fabricating a regular BH solution [49-51]. $\mathrm{He}$ and Lin [52] have investigated the deflection of light for test particles, due to a axially moving Kerr-Newman BH with an arbitrary constant velocity that is perpendicular to its angular momentum. Additionally, it is demonstrated that here is no peculiarity of the electric field quality at the cause for the point-like particles and it has an attractive charge. By using GBT, deflection angle of light on the foundation of magnetized BH and impact of non-linear electrodynamic has been found by Javed et al.[53,54]. Recently, plasma medium deflects photons shown by Crisnejo and Gallo [55]. By investigating the weak gravitational lensing for hairy BH in the back burner of Einstein-Maxwell theory (EMT) with a non-minimally coupled dilaton and its non-trivial potential, in this connection by virtue of GBT deflection angle of light has been computed with plasma and excluding plasma medium by Javed et al [56]. For more new works, one can see [57-86]. Main cause of this work is to study the stringy black holes by utilizing Gauss-Bonnet theorem and also to see the effect of topological defects on gravitational lensing. For examination, we consider the notation of the deflection angle of big particles and the deflection of photons in a plasma medium.

This paper arranged as: in section 2, We concisely review regarding stringy black holes. In section 3, we apply Gauss-Bonnet theorem to find deflection angle of stringy black holes. In section 4, we enhance our work to find out the deflection angle in the presence of plasma medium and in addition we demonstrate the graphical impact of deflection angle in the context of stringy black hole. In section 5, we recap our results which we obtain in present work.

\section{COMPUTATION OF WEAK LENSING BY STRINGY BLACK HOLES AND GAUSS-BONNET THEOREM}

The stringy black holes metric in spherically coordinate is given as [87];

$$
d s^{2}=\frac{d t^{2}}{F(r)^{2}}-F(r)^{2}\left\{d r^{2}+r^{2}\left(d \theta^{2}+\sin ^{2} \theta d \phi^{2}\right)\right\}
$$

where $F(r)$ is

$$
F(r)=1+\frac{Q}{r}+\frac{Q^{2} \alpha}{8 r(r+Q)^{3}} .
$$

The optical spacetime simply written in equatorial plane $\left(\theta=\frac{\pi}{2}\right)$ to get null geodesics $\left(d s^{2}=0\right)$

$$
d t^{2}=F(r)^{4} d r^{2}+r^{2} F(r)^{4} d \phi^{2} .
$$

The Gaussian optical curvature that is evaluated as follows:

$$
\mathcal{K}=\frac{\text { RicciScalar }}{2}
$$

After simplifying, Gaussian optical curvature is stated as:

$$
\mathcal{K} \approx-2 \frac{Q}{r^{3}}+\left(12 r^{-4}-4 \frac{\alpha}{r^{6}}\right) Q^{2}+\mathcal{O}\left(Q^{3}, \alpha^{2}, r^{7}\right)
$$

Now, we derive the deflection angle of a stringy BH by using the GBT. By applying the GBT to the region $\mathcal{N}_{R}$, given as [35]

$$
\int_{\mathcal{N}_{R}} \mathcal{K} d S+\oint_{\partial \mathcal{N}_{R}} k d t+\sum_{k} \sigma_{k}=2 \pi \mathcal{X}\left(\mathcal{N}_{R}\right)
$$

where, $k$ is geodesic curvature, $\mathcal{K}$ represent Gaussian curvature respectively, and $k$ is defined as $k=\bar{g}\left(\nabla_{\tilde{\alpha}} \tilde{\alpha}, \bar{\alpha}\right)$ in such a way that $\bar{g}(\tilde{\alpha}, \tilde{\alpha})=1$, here $\bar{\alpha}$ is unit acceleration vector and $\sigma_{k}$ represent the exterior angle of $k^{t h}$ vertex 
respectively. When $R \rightarrow \infty$ the jump angles equal to $\pi / 2$, hence $\sigma_{O}+\sigma_{S} \rightarrow \pi$. Where $\mathcal{X}\left(\mathcal{N}_{R}\right)=1$ is a Euler characteristic and $\mathcal{N}_{R}$ represents the non singular. Thus we obtian

$$
\iint_{\mathcal{N}_{R}} \mathcal{K} d S+\oint_{\partial \mathcal{N}_{R}} k d t+\sigma_{k}=2 \pi \mathcal{X}\left(\mathcal{N}_{R}\right) .
$$

Here, $\alpha_{\bar{g}}$ is a geodesic and the total jump angle is $\sigma_{k}=\pi$, since $\mathcal{X}$ represent Euler characteristic number which is 1 . When $R \rightarrow \infty$ then remaining part is $k\left(D_{R}\right)=\left|\nabla_{\dot{D}_{R}} \dot{D}_{R}\right|$. Considering the radial component for geodesic curvature that is described as:

$$
\left(\nabla_{\dot{D}_{R}} \dot{D}_{R}\right)^{r}=\dot{D}_{R}^{\phi} \partial_{\phi} \dot{D}_{R}^{r}+\Gamma_{\phi \phi}^{r}\left(\dot{D}_{R}^{\phi}\right)^{2}
$$

At very high $R, D_{R}:=r(\phi)=R=$ const. Thus, the leading term of above equation vanishes and $\left(\dot{D}_{R}^{\phi}\right)^{2}=\frac{1}{f^{2}\left(r^{\star}\right)}$. Recalling $\Gamma_{\phi \phi}^{r}=\frac{-2 r^{2} F^{\prime}}{F}-r$, we get

$$
\left(\nabla_{\dot{D}_{R}^{r}} \dot{D}_{R}^{r}\right)^{r} \rightarrow \frac{1}{R}
$$

And which proves that the geodesic curvature is not effected to the topological defects (i.e $\left.k\left(D_{R}\right) \rightarrow R^{-1}\right)$. We can write $d t=R d \phi$. Thus;

$$
k\left(D_{R}\right) d t=\frac{1}{R} R d \phi
$$

From the pervious results, we get

$$
\iint_{\mathcal{N}_{R}} \mathcal{K} d s+\oint_{\partial \mathcal{N}_{R}} k d t={ }^{R \rightarrow \infty} \iint_{S_{\infty}} \mathcal{K} d S+\int_{0}^{\pi+\sigma} d \phi
$$

In the weak field deflection limit at the zeroth order the light ray is described as $r(t)=b / \sin \phi$. Therefore, the deflection angle stated as: [35]

$$
\sigma=-\int_{0}^{\pi} \int_{b / \sin \phi}^{\infty} \mathcal{K} \sqrt{\operatorname{det} \bar{g}} d u d \phi
$$

We substitute the leading term of equation 21 into above equation 12, so the obtained deflection angle up to leading order term is stated as:

$$
\sigma \simeq \frac{4 Q}{b}-\frac{3 Q^{2} \pi}{b^{2}}+\frac{3 \pi Q^{2} \alpha}{8 b^{4}}+\mathcal{O}\left(Q^{3}, \alpha^{2}\right)
$$

\section{GRAPHICAL INFLUENCE OF DEFLECTION ANGLE UPON STRINGY BLACK HOLE}

In this portion, we analyze the graphical behavior of deflection angle $\sigma$ on stringy $\mathrm{BH}$. We also give the physical importance of these graphs to examine the effect of impact parameter $b$, coupling constant $\alpha$ and black holes charge $Q$ on deflection angle. 


\section{A. Deflection angle $\sigma$ w.r.t Coupling constat $\alpha$}

(i) $\alpha=10$

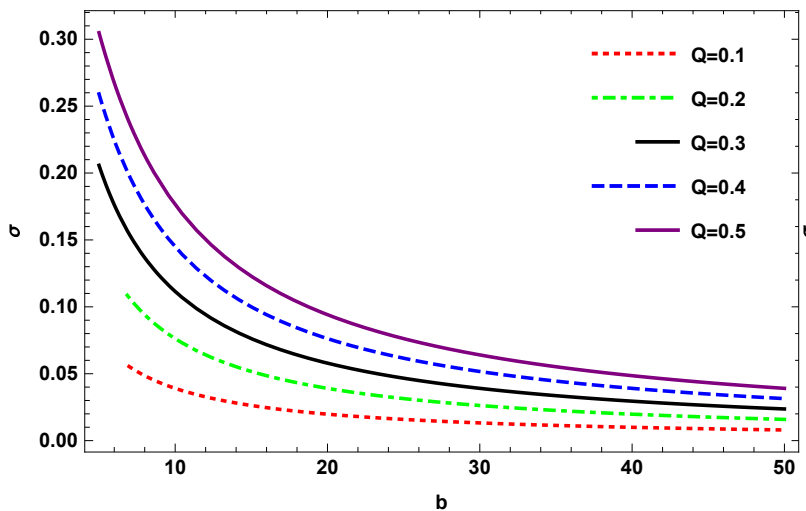

(i) $\alpha=10$

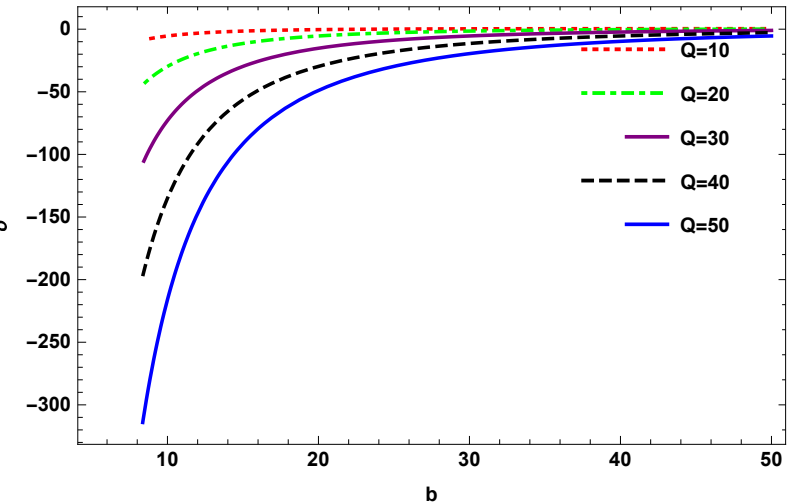

Figure 1: $\tilde{\alpha}$ versus $b$.

- Figure 1, the left plot shows the behavior of deflection angle $\sigma$ w.r.t $b$ for fixed BH charge $Q$ and varying $\alpha$ has been checked. We noticed that, for only negative values of $\alpha$ the behavior of deflection angle is stable but $\alpha>0$ indicates the unstable state of deflection angle.

The right plot renders the behavior of deflection angle $\sigma$ with $b$ by taking fixed $\alpha$ and varying $Q$. We analyzed that the deflection angle exponentially decreasing and approached to positive infinity. Thus the deflection angle get the stable behavior for positive values.

\section{WEAK LENSING BY STRINGY BLACK HOLES IN A PLASMA MEDIUM}

This segment is based on the investigation of weak gravitational lensing of Stringy black hole in the presence of plasma medium. For Stringy black holes the refractive index $n(r)$, is obtain as, [74]

$$
n(r)=\sqrt{1-\frac{\omega_{e}^{2}}{\omega_{\infty}^{2}} F(r)^{-2}}
$$

where $\omega_{e}$ and $\omega_{\infty}$ are electron plasma frequency and photon frequency measured by an observer at infinity respectively, then the corresponding optical metric illustrated as

$$
d t^{2}=g_{i j}^{o p t} d x^{i} d x^{j}=n^{2}(r)\left[F(r)^{4} d r^{2}+r^{2} F(r)^{4} d \phi^{2}\right]
$$

Here the metric function of the above optical metric is given as

$$
F(r)=1+\frac{Q}{r}+\frac{Q^{2} \alpha}{8 r(r+Q)^{3}} .
$$

Now, the value of Gaussian curvature is found as:

$$
\mathcal{K} \approx-2 \frac{Q}{r^{3}}+\left(12 r^{-4}-4 \frac{\alpha}{r^{6}}\right) Q^{2}+\frac{\omega_{e}^{2}}{\omega_{\infty}^{2}}\left(\frac{-3 Q}{2 r^{3}}+\frac{8 Q^{2}}{r^{4}}-\frac{3 Q^{2} \alpha}{r^{6}}\right)+\mathcal{O}\left(Q^{3}, \alpha^{2}\right) .
$$

For this, we use GBT to compute the deflection angle in order to relate it with non-plasma. As follows, for calculating angle in the weak field area, as the light beams become a straight line. Therefore, we use condition of $r=\frac{b}{\sin \varphi}$ at zero order.

$$
\sigma=-\lim _{R \rightarrow 0} \int_{0}^{\pi} \int_{\frac{b}{\sin \phi}}^{R} \mathcal{K} d S
$$

So, the deflection angle in the presence of plasma medium is defined as;

$$
\sigma \simeq \frac{4 Q}{b}-\frac{3 Q^{2} \pi}{b^{2}}+\frac{3 \pi Q^{2} \alpha}{8 b^{4}}+\frac{3 Q}{b} \frac{\omega_{e}^{2}}{\omega_{\infty}^{2}}+\frac{9 \pi Q^{2} \alpha}{32 b^{4}} \frac{\omega_{e}^{2}}{\omega_{\infty}^{2}}-\frac{2 Q^{2}}{b^{2}} \frac{\omega_{e}^{2}}{\omega_{\infty}^{2}}+\mathcal{O}\left(Q^{3}, \alpha^{2}\right)
$$

The above results tells us that photon rays are moving into medium of homogeneous plasma. We see that equation 19 reduced into equation 13 when plasma effect is removed. 


\section{GRAPHICAL ANALYSIS FOR PLASMA MEDIUM}

This section give us detailed graphical analysis of deflection angle $\sigma$ in the presence of plasma medium. We discussed the effect of black holes charge $Q$, impact parameter $b$ and $\beta$ on deflection angle for this, we take $\beta=$ $\frac{\omega_{e}^{2}}{\omega_{\infty}^{2}}=10^{-1}$

(ii) $\beta=10^{-1}, \alpha=6$

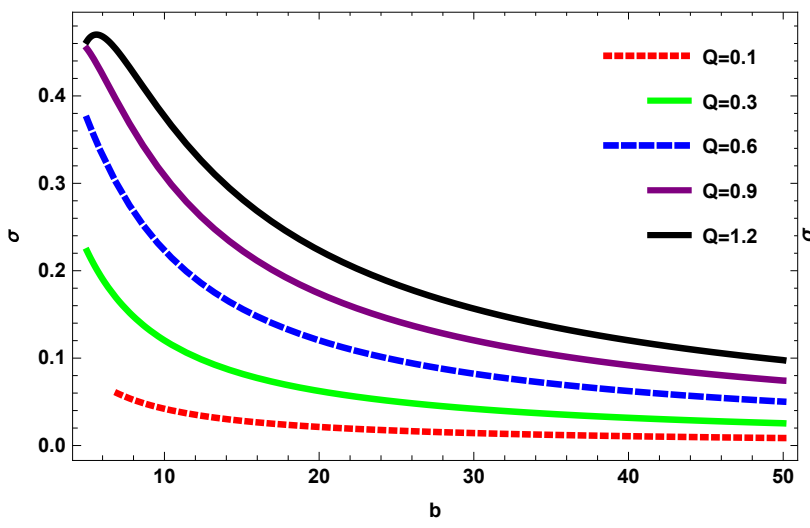

(ii) $Q=2.5, \alpha=6$

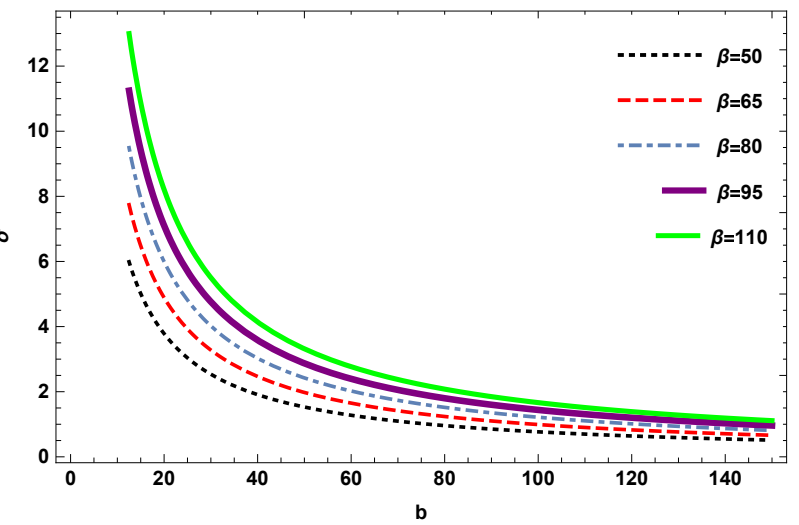

Figure 2: $\tilde{\alpha}$ versus $b$.

- Figure 2, the left plot provide us behavior of deflection angle $\sigma$ with impact parameter $b$ by changing value of $Q$ and taking fixed values of $\beta, \alpha$. We determine that for small values of $Q$ the deflection angle exponentially decreasing and then approached to positive infinity, but as we choose high values of $Q$ the behavior of deflection angle is unstable. Thus, we summarized that as we choose small values for $Q$ the behavior of deflection is stable. On contrary, as we choose high values for $Q$ the behavior of deflection angle is unstable.

The behaviors of deflection angle $\sigma$ with $b$ by changing the values of $\beta$ and for fixed $\alpha, Q$ has been analyzed in right plot. We examined that the deflection angle decreasing gradually and move towards positive infinity.

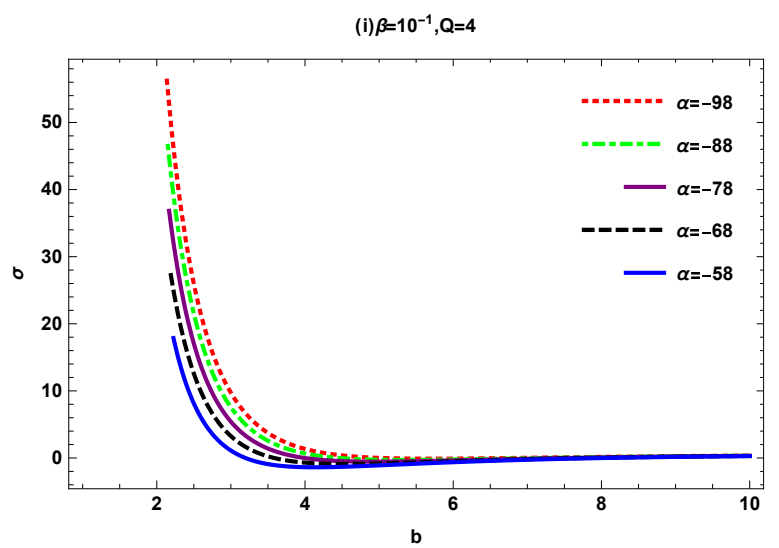

Figure 3: $\tilde{\alpha}$ versus $b$.

- Figure 3, Demonstrate the behavior of $\sigma$ with $b$ by changing the values of $\alpha$ and fixed $\beta$ and $Q$. We noticed that the behavior of deflection angle decreasing and eventually approached to positive infinity. The behavior of deflection angle decreasing negatively for $\alpha$ greater than zero.

\section{CONCLUSION}

The present paper is about the investigation of deflection angle by stringy BH in plasma and non-plasma medium. Initially, we study the weak gravitational lensing by using GBT and derived the deflection angle of photon. After 
that, our proposed deflection angle which is as follows,

$$
\sigma=\frac{4 Q}{b}+\frac{-Q^{2} \pi}{b^{2}}-\frac{3 Q^{2} \pi}{64 b^{4}}+\frac{3 Q \pi}{64 b^{4}}-\frac{32 Q^{2} \alpha}{75 b^{5}}+\mathcal{O}\left(Q^{3}, \alpha^{2}\right)
$$

We analyzed that by reduction of some parameters the obtained deflection angle converted into the Schwarzschild deflection angle. We also discuss the graphical effect of different parameters on deflection angle by stringy $\mathrm{BH}$. Deflection angle by stringy black holes w.r.t impact parameter:

We observed that, there is a direct relation between deflection angle angle and impact parameter while to obtain the stable behavior of deflection angle we only choose $0<Q \leq 1$ and negative $\alpha$.

We also observed that deflection angle in the presence of plasma medium given by Eq. (4.5). which is;

$$
\begin{aligned}
\sigma & \simeq \frac{4 Q}{b}-\frac{Q^{2} \pi}{b^{2}}+\frac{3 \alpha Q \pi}{64 b^{4}}+\frac{3 \alpha Q^{2} \pi}{64 b^{4}}-\frac{32 \alpha Q^{2}}{75 b^{5}}+\frac{Q}{b} \frac{\omega_{e}^{2}}{\omega_{\infty}^{2}} \\
& -\frac{11 Q^{2}}{8 b^{2}} \frac{\omega_{e}^{2}}{\omega_{\infty}^{2}}-\frac{7 \alpha Q^{2}}{b^{3}} \frac{\omega_{e}^{2}}{\omega_{\infty}^{2}}+\frac{2 Q}{b} \frac{\omega_{e}^{4}}{\omega_{\infty}^{4}}+\frac{62 Q^{2} \pi}{b} \frac{\omega_{e}^{4}}{\omega_{\infty}^{4}} \\
& +\frac{7 Q^{2} \pi}{8 b^{2}} \frac{\omega_{e}^{4}}{\omega_{\infty}^{4}}+\mathcal{O}\left(Q^{3}, \alpha^{2}\right) .
\end{aligned}
$$

If we neglected the term $\frac{\omega_{e}}{\omega_{\infty}} \rightarrow 0$ the plasma effect can be removed. Additionally, we demonstrate the graphical impact of deflection angle on stringy $\mathrm{BH}$ in a plasma medium.

We examined that the deflection angle $\sigma$ w.r.t $b$ can be analyzed only for negative large values of $\alpha$ to acquire the stable state of $\mathrm{BH}$. It is to be noticed that, for $\alpha>0$ and for negative small values of $\alpha$ the behavior of deflection angle is negatively decreasing which indicates the unstable state.

\section{Acknowledgments}

This work was supported by Comisión Nacional de Ciencias y Tecnología of Chile through FONDECYT Grant $N^{\circ}$ 3170035 (A. Ö.).

[1] B. P. Abbott et al. [LIGO Scientific and Virgo Collaborations], Phys. Rev. Lett. 116, no. 6, 061102 (2016).

[2] M. Barriola and A. Vilenkin, Phys. Rev. Lett. 63, 341 (1989).

[3] P. Jetzer, Phys. Rept. 220, 163 (1992).

[4] P. Jetzer and J. J. van der Bij, Phys. Lett. B 227, 341 (1989).

[5] R. D. Viollier, F. R. Leimgruber and D. Trautmann, Phys. Lett. B 297, 132 (1992).

[6] N. Bilic and R. D. Viollier, Phys. Lett. B 408, 75 (1997).

[7] H. Wei, D. C. Qiang, Z. X. Yu and H. K. Deng, arXiv:1911.04201 [gr-qc].

[8] J. Badia and E. F. Eiroa, Eur. Phys. J. C 77, no. 11, 779 (2017).

[9] D. C. Latimer, Phys. Rev. D 88, 063517 (2013)

[10] T. Elghozi, N. E. Mavromatos and M. Sakellariadou, Eur. Phys. J. C 77, no. 7, 445 (2017)

[11] B. Ahmedov, B. Turimov, Z. Stuchlík and A. Tursunov, Int. J. Mod. Phys. Conf. Ser. 49, 1960018 (2019).

[12] B. Turimov, B. Ahmedov, A. Abdujabbarov and C. Bambi, arXiv:1802.03293 [gr-qc].

[13] A. Abdujabbarov, B. Ahmedov, N. Dadhich and F. Atamurotov, Phys. Rev. D 96, no. 8, 084017 (2017).

[14] J. Schee, Z. Stuchlík, B. Ahmedov, A. Abdujabbarov and B. Toshmatov, Int. J. Mod. Phys. D 26, no. 5, 1741011 (2017).

[15] H. Ghaffarnejad, M. Amirmojahedi and H. Niad, Adv. High Energy Phys. 2018, 3067272 (2018)

[16] A. B. Aazami, C. R. Keeton and A. O. Petters, J. Math. Phys. 52, 102501 (2011)

[17] K. S. Virbhadra and C. R. Keeton, Phys. Rev. D 77, 124014 (2008)

[18] C. R. Keeton and A. O. Petters, Phys. Rev. D 73, 044024 (2006)

[19] C. R. Keeton and A. O. Petters, Phys. Rev. D 72, 104006 (2005)

[20] A. Bhadra, Phys. Rev. D 67, 103009 (2003)

[21] W. G. Cao and Y. Xie, Eur. Phys. J. C 78, no. 3, 191 (2018).

[22] Y. K. Lim and Q. h. Wang, Phys. Rev. D 95, no. 2, 024004 (2017)

[23] J. Sultana, JCAP 1304, 048 (2013).

[24] P. Fleury, J. Larena and J. P. Uzan, Phys. Rev. Lett. 119, no. 19, 191101 (2017)

[25] R. Whisker, Phys. Rev. D 71, 064004 (2005)

[26] S. b. Chen and J. 1. Jing, Phys. Rev. D 80, 024036 (2009) 
[27] E. F. Eiroa, G. E. Romero and D. F. Torres, Phys. Rev. D 66, 024010 (2002)

[28] C. Y. Wang, Y. F. Shen and Y. Xie, JCAP 1904, 022 (2019)

[29] S. Mao and B. Paczynski, Astrophys. J. 374, L37 (1991).

[30] V. Bozza, Phys. Rev. D 66, 103001 (2002)

[31] M. Sharif and S. Iftikhar, Astrophys. Space Sci. 357, no. 1, 85 (2015).

[32] K. S. Virbhadra and G. F. R. Ellis, Phys. Rev. D 65, 103004 (2002).

[33] O. Kasikci and C. Deliduman, Phys. Rev. D 100, no. 2, 024019 (2019)

[34] R. Zhang and J. Jing, Eur. Phys. J. C 78, no. 10, 796 (2018).

[35] G. W. Gibbons and M. C. Werner, Class. Quant. Grav. 25, 235009 (2008).

[36] M. C. Werner, Gen. Relat. gravit. 44, 3047 (2012).

[37] H. Arakida and M. Kasai, Phys. Rev. D 85, 023006 (2012).

[38] G. S. Bisnovatyi-Kogan and O. Y. Tsupko, Universe 3, no. 3, 57 (2017).

[39] K. Jusufi, A. Ovgun, Phys. Rev. D 97(2), 024042 (2018).

[40] K. Jusufi, A. Övgün, A. Banerjee and I. Sakalli, Eur. Phys. J. Plus 134, no. 9, 428 (2019).

[41] A. Övgün, K. Jusufi and I. Sakalli, Phys. Rev. D 99, 024042 (2019).

[42] A. Övgün, Phys. Rev. D 98, 044033 (2018).

[43] A. Övgün, K. Jusufi, and I. Sakalli, Annals Phys. 399, 193 (2018).

[44] A. Övgün, K. Jusufi, and I. Sakalli, Annals Phys. 399, 193 (2018).

[45] A. Övgün, Universe 5, 115 (2019).

[46] W. Javed, R. Babar, A. Ovgun, Phys. Rev. D 99(8), 084012 (2019).

[47] M. S. Ma, Annals Phys. 362, 529 (2015)

[48] O. Miskovic and R. Olea, Phys. Rev. D 83, 024011 (2011)

[49] O. Miskovic and R. Olea, Phys. Rev. D 83, 064017 (2011)

[50] B. Toshmatov, Z. Stuchlík, J. Schee and B. Ahmedov, Phys. Rev. D 97, no. 8, 084058 (2018)

[51] K. A. Bronnikov, Phys. Rev. D 63, 044005 (2001)

[52] G. He and W. Lin, Class. Quant. Grav. 33, no. 9, 095007 (2016) Addendum: [Class. Quant. Grav. 34, no. 2, 029401 (2017)].

[53] W. Javed, A. Hazma and A. Övgün, Preprints 2019, 2019110142 (doi:10.20944/preprints201911.0142.v1)

[54] W. Javed, J. Abbas, and A. Övgün, Eur. Phys. J. C 79, no. 8, 694 (2019).

[55] G. Crisnejo and E. Gallo, Phys. Rev. D 97, no. 12, 124016 (2018)

[56] W. Javed, j. Abbas and A. Övgün, Phys. Rev. D 100, no. 4, 044052 (2019).

[57] A. Ishihara, Y. Suzuki, T. Ono, T. Kitamura and H. Asada, Phys. Rev. D 94, no. 8, 084015 (2016)

[58] A. Ishihara, Y. Suzuki, T. Ono and H. Asada, Phys. Rev. D 95, no. 4, 044017 (2017).

[59] H. Arakida, Gen. Rel. Grav. 50, no. 5, 48 (2018).

[60] K. Jusufi, M.C. Werner, A. Banerjee, A. Ovgun, Phys. Rev. D 95(10), 104012 (2017).

[61] I. Sakalli, A. Ovgun, EPL 118(6), 60006 (2017).

[62] A. Övgün, Phys. Rev. D 99, 104075 (2019).

[63] T. Ono, A. Ishihara and H. Asada, Phys. Rev. D 96, 104037 (2017).

[64] K. Jusufi, A. Övgün and A. Banerjee, Phys. Rev. D 96, n084036 (2017) Addendum: [Phys. Rev. D 96, 089904 (2017)].

[65] T. Ono, A. Ishihara, and H. Asada, Phys. Rev. D 99, no. 12, 124030 (2019).

[66] K. Jusufi and A. Övgün, Int. J. Geom. Meth. Mod. Phys. (2019) 1950116.

[67] T. Ono, A. Ishihara and H. Asada, Phys. Rev. D 98, 044047 (2018).

[68] K. Jusufi, M. C. Werner, A. Banerjee, and A. Övgün, Phys. Rev. D 95, no. 10, 104012 (2017).

[69] Z. Li and T. Zhou, arXiv:1908.05592 [gr-qc].

[70] K. Jusufi, I. Sakalli, and A. Övgün, Phys. Rev. D 96, no. 2, 024040 (2017).

[71] Z. Li, G. He and T. Zhou, arXiv:1908.01647 [gr-qc].

[72] K. Jusufi, A. Övgün, J. Saavedra, Y. Vasquez, and P. A. Gonzalez, Phys. Rev. D 97, 124024 (2018).

[73] K. de Leon and I. Vega, Phys. Rev. D 99, no. 12, 124007 (2019)

[74] G. Crisnejo, E. Gallo, and A. Rogers, Phys. Rev. D 99, 124001 (2019).

[75] K. Jusufi and A. Övgün, Phys. Rev. D 97, 064030 (2018).

[76] G. Crisnejo, E. Gallo, and J. R. Villanueva, Phys. Rev. D 100, no. 4, 044006 (2019).

[77] A. Övgün, G. Gyulchev, and K. Jusufi, Annals Phys. 406, 152 (2019).

[78] A. Övgün, I. Sakalli, and J. Saavedra, Annals Phys. 411, 167978 (2019).

[79] A. Övgün, I. Sakalli, and J. Saavedra, JCAP 1810, 041 (2018).

[80] W. Javed, R. Babar, and A. Övgün, Phys. Rev. D 100, 104032 (2019).

[81] W. Javed, J. Abbas and A. Övgün, Preprints 2019, 2019060124. (doi: 10.20944/preprints201906.0124.v1).

[82] W. Javed, j. Abbas and A. Övgün, Phys. Rev. D 100, no. 4, 044052 (2019).

[83] Y. Kumaran and A. Övgün, arXiv:1905.11710 [gr-qc].

[84] W. Javed, J. Abbas, Y. Kumaran and A. Övgün, Preprints 2019, 2019110210 (doi: 10.20944/preprints201911.0210.v1).

[85] Z. Li and A. Övgün, Preprints 2019, 2019110195 (doi: 10.20944/preprints201911.0195.v1)

[86] A. Övgün, I. Sakalli and J. Saavedra, arXiv:1908.04261 [gr-qc].

[87] P. A. Cano, T. Ortín and P. F. Ramirez, arXiv:1909.08530 [hep-th]. 